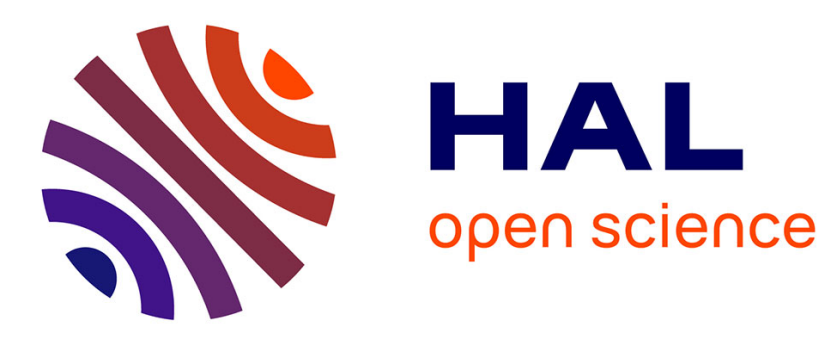

\title{
Aircraft fault tolerant control based on active set method
}

\author{
Lunlong Zhong, Felix Mora-Camino
}

\section{To cite this version:}

Lunlong Zhong, Felix Mora-Camino. Aircraft fault tolerant control based on active set method. CCDC 2013, 25th Chinese Control and Decision Conference, May 2013, Guiyang, China. pp 35163521 ; ISBN : 978-1-4673-5533-9, 10.1109/CCDC.2013.6561557 . hal-00926493

\section{HAL Id: hal-00926493 \\ https://hal-enac.archives-ouvertes.fr/hal-00926493}

Submitted on 14 Jan 2014

HAL is a multi-disciplinary open access archive for the deposit and dissemination of scientific research documents, whether they are published or not. The documents may come from teaching and research institutions in France or abroad, or from public or private research centers.
L'archive ouverte pluridisciplinaire HAL, est destinée au dépôt et à la diffusion de documents scientifiques de niveau recherche, publiés ou non, émanant des établissements d'enseignement et de recherche français ou étrangers, des laboratoires publics ou privés. 


\title{
Aircraft Fault Tolerant Control Based on Active Set Method
}

\author{
Lunlong Zhong ${ }^{1,2}$, Félix Mora-Camino ${ }^{1,2}$ \\ 1. ENAC, MAIAA, F-31055 Toulouse, France \\ 2. Univ de Toulouse, F-31400 Toulouse, France \\ E-mail: lunlong.zhong@enac.fr, felix.mora@enac.fr
}

\begin{abstract}
This communication considers the case in which an aerodynamic actuator failure occurs to an aircraft while it has to perform a guidance manoeuver. The problem considered deals with the reassignment of the remaining actuators to continue to perform the manoeuver while maintaining the structural integrity of the aircraft. A nonlinear inverse control technique is used to generate online nominal moments along the three main axes of the aircraft. Then, taking into account all material and structural constraints as well as the redundant effects from other actuators, a Mathematical Programming problem to be solved online is introduced. The proposed on line solution method is based on an active set method which appears to provide acceptable response times according to the simulation results. Then new development perspectives are discussed.
\end{abstract}

Key Words: Fault tolerant control, Flight mechanics, Quadratic programming, Active set method, Actuators assignment

\section{INTRODUCTION}

In this paper we consider a transportation airplane in the situation in which a main aerodynamic actuator failure occurs while it has to perform guidance manoeuvers. Here using dynamic inversion of flight dynamics, the necessary moments to perform a given guidance manoeuver are computed, and then an optimization problem is considered to generate on-line reference values for the actuators. This represents the main difference with other previous approaches to actuator fault management [1-3]. In the case considered here, a linear quadratic programming formulation of the optimization problem can be adopted and an active set approach to get an on-line solution is discussed. The approach is also different from the one we proposed [4] before which is based on a neural network dynamic solver.

\section{Effectiveness of Aerodynamic Actuators}

The effectiveness of the control surfaces appears through the contributions of their angular deflections to the dimensionless coefficients present in the expressions of aerodynamic forces and torques [5]. These control surfaces produce as well a collective external effect over the whole aircraft as internal efforts which should satisfy structural constraints. The global dimensionless coefficients used to express aerodynamic forces are assumed to be given by:

$$
\begin{gathered}
C_{x}=C_{x 0}+k C_{z}^{2} \\
C_{y}=C_{y \beta} \beta+C_{y p} p l_{A} / V+C_{y r} r l_{A} / V+\underline{C}_{y \delta p}{ }^{\prime} \underline{\delta}_{p}+\underline{C}_{y} \delta_{r}{ }^{\prime} \underline{\delta}_{r}(1 \\
C_{z}=C_{z 0}+C_{z \alpha} \alpha+C_{z \delta t h s} \delta_{t h s}+\underline{C}_{z} \delta q
\end{gathered}
$$

where $k$ is a positive coefficient and the $C_{i j}$ are dimensionless aerodynamic derivatives. Here $p, q, r$ are respectively the roll, pitch and yaw rates, $\alpha$ is the angle of attack, $\beta$ is the side slip angle, $\delta_{p}, \delta_{q}, \delta_{r}$ are respectively the aileron, elevator and rudder deflections.

The dimensionless coefficients of the main axis aerodynamic torques can in general be expressed such as:

$$
\begin{gathered}
C_{m}=C_{m 0}+C_{m \alpha} \alpha+C_{m q} q l_{A} / V+C_{m \delta t h s} \delta_{t h s}+\underline{C}_{m \delta q}{ }^{\prime} \underline{\delta}_{q} \\
C_{l}=C_{l 0}+C_{l \beta} \beta+C_{l p} p l_{A} / V+C_{l r} r l_{A} / V+\underline{C}_{l \delta p} \underline{\delta}_{p}+\underline{C}_{l \delta r}{ }^{\prime} \underline{\delta}_{r} \\
C_{n}=C_{n 0}+C_{n \beta} \beta+C_{n p} p l_{A} / V+C_{n r} r l_{A} / V+\underline{C}_{n \delta p}, \underline{\delta}_{p}+\underline{C}_{n \delta r}{ }^{\prime} \underline{\delta}_{r}
\end{gathered}
$$

where $V$ is the airspeed, $\delta_{\text {ths }}$ is the angular position of the trimmable horizontal stabilizer and $l_{A}$ is a reference length. Then the expression of the different aerodynamic torques generated by the control surfaces can be approximated by an affine form with respect to the corresponding deflections of the different aerodynamic actuators, so that we get expressions such as:

$$
M_{i k}=M_{i k}^{0}+\mu_{i k} \delta_{k}
$$

where $M_{i k}$ is the $i^{\text {th }}$ considered moment (roll, pitch, yaw, bending, twisting), $\delta_{k}$ is the deflection of the $k^{\text {th }}$ aerodynamic actuator $(k \in K=\{$ aileron, flap, right spoilers, left spoilers, elevator, rudder $\}$ ) and $\mu_{i k}$ is the current effectiveness of actuator $k$ to produce moment $i$. The current values $M_{i k}{ }^{0}(\mathrm{t})$ and $\mu_{i k}(\mathrm{t})$ depend on the airspeed, the flight level and on the values of $\alpha, \beta, p, q$ and $r$. Global aerodynamic torques generated by aircraft aerodynamic actuators can be rewritten in a global affine form as:

$$
\begin{gathered}
L(t)=L^{0}(t)+\sum_{i \in I^{L}} X_{i}^{L}(t) \delta_{i}(t) \\
M(t)=M^{0}(t)+\sum_{i \in I^{M}} X_{i}^{M}(t) \delta_{i}(t) \\
N(t)=N^{0}(t)+\sum_{i \in I^{N}} X_{i}^{N}(t) \delta_{i}(t)
\end{gathered}
$$

with $I=I^{L} \cup I^{M} \cup I^{N}$, where $I^{L}$ is the set of actuators generating some roll moment, $I^{N}$ is the set of actuators 
generating some yaw torque, while $I^{M}$ is the set of actuators generating pitch moments. Fig 1 displays, in the case of a A340 aircraft, the different aerodynamic surfaces of its wing. The current values of $L^{0}(t), X_{i}^{L}(t), M^{0}(t)$, $X_{i}^{M}(t), N^{0}(t)$ and $X_{i}^{N}(t)$ depend on the airspeed, the flight level and $\alpha, \beta, p, q$ and $r$.

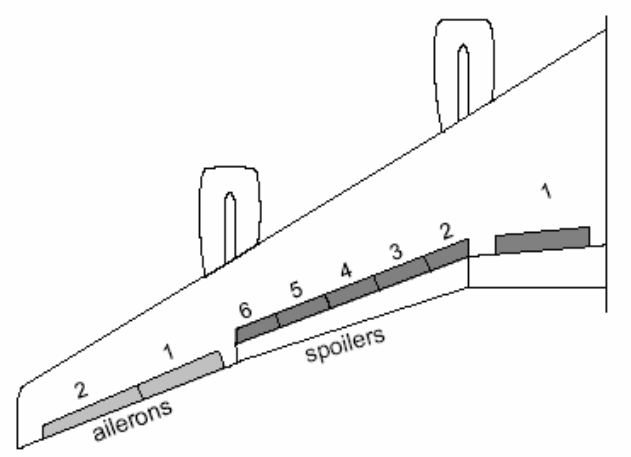

Fig 1. Aerodynamic surfaces of A340 wing

\section{Actuators constraints and limitations}

The deflection of each aerodynamic surface is subject to minimum and maximum bounds while its deflection rates are limited by the adopted actuator technology. Also, global physical constraints must be taken into account to insure aircraft integrity. These limitations should be taken explicitly into consideration by the reassignment system.

\subsection{Actuators position and speed limitations}

With respect to control surfaces, the following bound constraints should be met:

$$
\begin{array}{ll}
\delta_{i}^{\min } \leq \delta_{i} \leq \delta_{i}^{\max } & i \in I \\
\dot{\delta}_{i}^{\min } \leq \dot{\delta}_{i} \leq \dot{\delta}_{i}^{\max } & i \in I
\end{array}
$$

where $\delta_{i}^{\min }, \delta_{i}^{\max }, \dot{\delta}_{i}^{\min }$ and $\dot{\delta}_{i}^{\max }$ are the bounds and maximum deflection speed values. These conditions can be considered at sampled instants, it becomes:

$$
\max \left\{\delta_{i}^{\min }, \delta_{i}(t-\Delta t)+\dot{\delta}_{i}^{\min } \Delta t\right\} \leq \delta_{i}(t)
$$

and

$$
\delta_{i}(t) \leq \min \left\{\delta_{i}^{\max }, \delta_{i}(t-\Delta t)+\dot{\delta}_{i}^{\max } \Delta t\right\}
$$

\subsection{Global constraints}

Global constraints are in general related with structural considerations. It has been shown in [6] that total wing bending and twisting torques during manoeuver can be written in an affine form as:

$$
M_{b}(t)=A_{b}(t)+\sum_{i \in I^{\text {wing }}} Y_{b i}(t) \delta_{i}(t)
$$

and

$$
M_{f}(t)=A_{f}(t)+\sum_{i \in I^{\text {wing }}} Y_{f i}(t) \delta_{i}(t)
$$

with $I^{\text {wing }} \subset I$ is the set of wing actuators contributing to the bending and the twisting torques, where $A_{b}, Y_{b i}, A_{f}$ and
$Y_{f i}$ depend also on the airspeed, the flight level and $\alpha, \beta, p, q$ and $r$.

Then the global wing bending and twisting constraints can be written as:

$$
A_{b}(t)+\sum_{i \in I^{\text {wing }}} Y_{b i}(t) \delta_{i}(t) \leq M_{b e n d}^{\max }
$$

and

$$
A_{f}(t)+\sum_{i \in T^{\text {wing }}} Y_{f i}(t) \delta_{i}(t) \leq M_{f l e x}^{\max }
$$

where $M_{\text {bend }}^{\max }$ and $M_{\text {flex }}^{\max }$ are maximum acceptable bending and twisting torques at the wing root. Here it is supposed that the satisfaction of these global constraints implies the satisfaction of local bending and twisting torque constraints.

\section{Optimization Problem Formulation for Actuators Reassignment}

To illustrate the proposed approach, here we consider the case of a pure stabilized roll manoeuver where the following conditions should be met by the body angular rates of the aircraft:

$$
\begin{gathered}
\tau_{p} \dot{p}+p=p_{c} \\
q=0 \\
\tau_{r} \dot{r}+r=(g / V) \sin \phi
\end{gathered}
$$

There roll and yaw motions follow first order dynamics while pitch dynamics remains frozen. Here $p_{c}$ is the desired roll rate and $\tau_{p}$ and $\tau_{r}$ are time constants. The dynamic constraint relative to the yaw rate is characteristic of a coordinated turn and its completion should allow avoiding noticeable lateral load factors during this roll manoeuver.

Applying the non linear inverse control approach [7], we get the necessary on-line values for each aerodynamic torque:

$$
\tilde{M}(t)=(A-C) r(t) p(t)+E\left(p(t)^{2}-r(t)^{2}\right)
$$

and

$$
\left[\begin{array}{c}
\tilde{L}(t) \\
\tilde{N}(t)
\end{array}\right]=\left[\begin{array}{cc}
A & -E \\
-E & C
\end{array}\right]\left[\begin{array}{c}
\frac{1}{\tau_{p}}\left(p_{c}-p(t)\right) \\
\frac{1}{\tau_{r}}((g / V(t)) \sin \phi(t)-r(t))
\end{array}\right]
$$

Here we consider the situations where the failure affects some of the commonly used actuators but the designed actuator redundancy still allows performing some manoeuvers.

Depending on the remaining degree of redundancy between elementary actuators, it may be possible to find a solution matching exactly the following moment constraints:

$$
\begin{gathered}
\sum_{i \in I^{L}} X_{i}^{L}(t) \tilde{\delta}_{i}(t)=\tilde{L}(t)-L^{0}(t) \\
\sum_{i \in I^{M}} X_{i}^{M}(t) \tilde{\delta}_{i}(t)=\tilde{M}(t)-M^{0}(t) \\
\sum_{i \in I^{N}} X_{i}^{N}(t) \tilde{\delta}_{i}(t)=\tilde{N}(t)-N^{0}(t)
\end{gathered}
$$


In this case the manoeuver will be performed still in a standard way. Otherwise, an approximate manoeuver should be defined.

In order to get a feasible reassignment avoiding fast and large solicitations of the actuators which could activate some structural modes of the aircraft, solutions as close as possible to the solution at the previous instant will be privileged. Also, it is admitted that when the standard manoeuver cannot be no more performed, close manoeuvers, in fact slightly downgraded manoeuvers, will be retained as a running solution. So, instead of considering the pure satisfaction of the moment constraints (11.1), (11.2) and (11.3) a measure $m(\underline{\tilde{\delta}}, \widetilde{L}, \tilde{M}, \tilde{N})$ of the degree of satisfaction of these constraints is introduced. Here we propose to choose the following measure:

$$
\begin{array}{r}
m(\underline{\tilde{\delta}}, \tilde{L}, \tilde{M}, \tilde{N})=w_{L}\left(\sum_{i \in I^{L}} X_{i}^{L}(t) \tilde{\delta}_{i}(t)-\tilde{L}(t)+L^{0}(t)\right)^{2} \\
+w_{M}\left(\sum_{i \in I^{M}} X_{i}^{M}(t) \tilde{\delta}_{i}(t)-\tilde{M}(t)+M^{0}(t)\right)^{2} \\
+w_{N}\left(\sum_{i \in I^{N}} X_{i}^{N}(t) \tilde{\delta}(t)-\tilde{N}(t)+N^{0}(t)\right)^{2}
\end{array}
$$

Then here our purpose is to solve on-line a linear quadratic optimization problem minimizing the following objective function:

$$
J(\underline{\tilde{\delta}})=\sum_{i \in I} \pi_{i} \cdot\left(\tilde{\delta}_{i}(t)-\tilde{\delta}_{i}(t-\Delta t)\right)^{2}+\gamma \cdot m(\underline{\tilde{\delta}}, \tilde{L}, \tilde{M}, \tilde{N})
$$

where the $\pi_{i}, i \in I$ and $\gamma$ are positive weights.

The complete definition of this optimization problem is such as:

$$
\min _{\underline{\tilde{\delta}}} J(\underline{\widetilde{\delta}})
$$

with the following structural constraints:

$$
\begin{aligned}
& A_{b}(t)+\sum_{i \in I^{\text {wing }}} Y_{b i}(t) \tilde{\delta}_{i}(t) \leq M_{\text {bend }}^{\max } \\
& A_{f}(t)+\sum_{i \in I^{\text {wing }}} Y_{f i}(t) \tilde{\delta}_{i}(t) \leq M_{\text {flex }}^{\max }
\end{aligned}
$$

and with the box constraints:

$$
\begin{gathered}
\max \left\{\delta_{i}^{\min }, \tilde{\delta}_{i}(t-\Delta t)+\dot{\delta}_{i}^{\min } \Delta t\right\} \leq \tilde{\delta}_{i}(t) \quad i \in I_{\bar{F}} \\
\tilde{\delta}_{i}(t) \leq \min \left\{\delta_{i}^{\max }, \tilde{\delta}_{i}(t-\Delta t)+\dot{\delta}_{i}^{\max } \Delta t\right\} \quad i \in I_{\bar{F}} \\
\max \left\{\tilde{\delta}_{i}^{\min }, \tilde{\delta}_{i}(t-\Delta t)+\dot{\delta}_{i}^{\min } \Delta t\right\} \leq \tilde{\delta}_{i}(t) \quad i \in I_{F L} \\
\tilde{\delta}_{i}(t) \leq \min \left\{\tilde{\delta}_{i}^{\max }, \tilde{\delta}_{i}(t-\Delta t)+\dot{\delta}_{i}^{\max } \Delta t\right\} \quad i \in I_{F L} \\
\max \left\{\tilde{\delta}_{i}^{\min }, \tilde{\delta}_{i}(t-\Delta t)+\tilde{\dot{\delta}}_{i}^{\min } \Delta t\right\} \leq \tilde{\delta}_{i}(t) \quad i \in I_{F S} \\
\tilde{\delta}_{i}(t) \leq \min \left\{\tilde{\delta}_{i}^{\max }, \tilde{\delta}_{i}(t-\Delta t)+\tilde{\dot{\delta}}_{i}^{\max } \Delta t\right\} \quad i \in I_{F S}
\end{gathered}
$$

with

$$
\begin{gathered}
\widetilde{\delta}_{i_{j}}=0 \text { if } i_{j} \in I_{F F}, j \in\{p, q, r, t h s\} \\
\widetilde{\delta}_{i_{j}}=\bar{\delta}_{i_{j}} \text { if } i_{j} \in I_{F P}, j \in\{p, q, r, t h s\}
\end{gathered}
$$

where $I_{\bar{F}}$ is the set of fully operational actuators, $I_{F L}, I_{F S}$ are respectively the set of actuators whose angular positions, angular speed are subject to additional limitations, $I_{F P}$ is the set of actuators which are stuck at a known angular position, $I_{F F}$ is the set of actuators which are not subject to a torque from their servo-control and with a zero deflection. The positive parameters $w_{L}, w_{M}$ and $w_{N}$ are chosen in the case of a roll manoeuver such as:

$$
w_{L}>>w_{M} \text { and } w_{L}>>w_{N}
$$

The above mathematical programming problem can be solved using standard programming techniques and making use as a start of the previous value of the deflections of the actuators. Then in a few iterations the solution of this small size linear quadratic problem should be obtained.

\section{Active Set Method Applied to Linear Quadratic Optimization Problems}

Active set algorithm is a name for a family of methods used to solve optimization problems with equality/inequality constraints. The idea underlying active set methods is to generate successive partitions of the inequality constraints into two groups: those that are to be treated as active and those that are to be treated as inactive. The method reduces equality/inequality constrained problem to a sequence of equality-only constrained sub-problems. At each iteration, active inequality constraints are treated as equality ones, they constitute at that time the working set, inactive ones are temporarily ignored and used afterwards to update the solution and working set. For a review, see [8], [9].

Problem (14-17) can be rewritten as a general quadratic programming problem as follows:

$$
\begin{array}{cc}
\min _{\underline{\delta}} & f(\underline{\delta})=\frac{1}{2} \underline{\delta}^{T} Q \underline{\delta}+\underline{c}^{T} \underline{\delta} \\
\text { s.t. } & g(\underline{\delta})=A \underline{\delta}-\underline{b} \leq \underline{0} \\
& \underline{\xi}^{-} \leq \underline{\delta} \leq \underline{\xi}^{+}
\end{array}
$$

where $\underline{\delta}$ can be the actuator deflections vector and matrix $Q$ is assumed symmetric positive definite. It has been already proved that the active set method solves problems such as $(19,20)$ after a finite number of iterations [8].

Here bound limits are taken as two different inequalities and problem $(19,20)$ can be written as:

$$
\begin{array}{cc}
\min _{\underline{\delta}} f(\underline{\delta})=\frac{1}{2} \underline{\delta}^{T} Q \underline{\delta}+\underline{c}^{T} \underline{\delta} \\
\text { s.t. } \quad g(\underline{\delta})=\tilde{A} \underline{\delta}-\underline{\tilde{b}} \leq \underline{0}
\end{array}
$$

where $\tilde{A}=\left[\begin{array}{c}A \\ I_{d} \\ -I_{d}\end{array}\right], \underline{\tilde{b}}=\left[\begin{array}{c}\underline{b} \\ \underline{\xi}^{+} \\ -\underline{\xi}^{-}\end{array}\right], I_{d}$ is an identity matrix of size $|\mathrm{I}| \times|\mathrm{I}|$.

At each iteration, the active set method solves a sub-problem (equality constrained QP). i.e.

$$
\begin{aligned}
& \min _{\underline{\delta}} f_{w}(\underline{\delta})=\frac{1}{2} \underline{\delta}^{T} Q \underline{\delta}+\underline{c}^{T} \underline{\delta} \\
& \text { s.t. } \quad \tilde{A}_{w} \underline{\delta}-\underline{\tilde{b}}_{w}=\underline{0}
\end{aligned}
$$


The subscript $w$ denotes the working set index. The solution at $k^{\text {th }}$ iteration is written $\underline{\delta}_{k}$. Let $\underline{p}$ be the solution of problem $(23,24)$ at iteration $k$. Then we have

$$
\begin{array}{ll}
\min _{\underline{p}} & f_{w}(\underline{p})=\frac{1}{2} \underline{p}^{T} Q \underline{p}+\underline{p}^{T}\left(Q \underline{\delta}_{k}+\underline{c}\right) \\
\text { s.t. } \quad \tilde{A}_{w} \underline{p}_{k+1}=\underline{0}
\end{array}
$$

Consider the KKT necessary and sufficient optimality conditions [10] of problem $(25,26)$.

$$
\left[\begin{array}{cc}
Q & \tilde{A}_{w}^{T} \\
\tilde{A}_{w} & 0
\end{array}\right]\left\{\begin{array}{l}
\underline{p} \\
\underline{\lambda}
\end{array}\right\}=\left\{\begin{array}{c}
-\left(Q \underline{\delta}_{k}+\underline{c}\right) \\
\underline{0}
\end{array}\right\}
$$

As long as $\tilde{A}_{w}$ is full row-rank, and since $Q$ is a positive definite matrix in our case, the KKT matrix in (27) is nonsingular [11]. Then Solving (27) is straightforward and we get a new search direction $\underline{p}_{k+1}$ and the associated Lagrange multipliers $\lambda_{k+1}$. According to their values, the current solution can be optimal; otherwise we get a new search direction.

When the current solution needs to be updated, the corresponding step length can be derived from a line search process:

$$
\underline{\delta}_{k+1}=\underline{\delta}_{k}+\alpha_{k+1} \underline{p}_{k+1}
$$

To make sure that $\delta_{k+1}$ is feasible, we only need to consider the constraints that are not in the working set and such as $\tilde{A}_{i} \underline{p}_{k+1}>0$, while we want $\alpha_{k+1}$ to be as large as possible within [0,1], so $\alpha_{k+1}$ is given by:

$$
\alpha_{k+1} \triangleq \min \left\{1, \min _{i \notin w_{k}, \tilde{A}_{i} \underline{p}_{k+1}>0}\left(\frac{\tilde{b}_{i}-\tilde{A}_{i} \underline{\delta}_{k}}{\tilde{A}_{i} \underline{p}_{k+1}}\right)\right\}
$$

Based on [9], we state here an algorithm to solve problem $(21,22)$ according to the active set approach:

\section{Algorithm:}

Compute or take the previous solution as a feasible starting point $\underline{\delta_{0}}$;

Let $W_{0}$ be the working set corresponding to $\underline{\delta}_{0}$;

for $\mathrm{k}=0,1,2, \ldots$

$$
\begin{aligned}
& \text { solve (27) to find } \underline{p}_{k+1} \text { and } \underline{\lambda}_{k+1} ; \\
& \text { if } \underline{p}_{k+1}=\underline{0} \\
& \text { if } \underline{\lambda}_{k+1}>=0 \\
& \text { STOP with solution } \underline{\delta}^{*}=\underline{\delta}_{\mathrm{k}} ; \\
& \text { else } \\
& \text { set } j=\arg \min \underline{\lambda}_{k} \\
& \underline{\delta}_{k+1}=\underline{\delta}_{k} ; W_{k+1}=W_{k}-\{\mathrm{j}\} \\
& \text { else }\left(\underline{p}_{k} \neq 0\right) \\
& \text { compute } \alpha_{k+1} \text { from }(28) \\
& \underline{\delta_{k+1}}=\underline{\delta}_{k}+\alpha_{k+1} p_{k+1} ; \\
& \text { if there are new active constraints } \\
& \text { add one of them to } W_{k+1} ; \\
& \text { else }
\end{aligned}
$$$$
\text { end (for) }
$$

In the case in which there are only bound limits, the resolution of (27) and the above proposed algorithm can be simplified as in [12].

\section{Application of the Active Set Method to Optimal Actuators Reassignment Problem}

This example is taken from [13], which is concerned with the control of the unstable lateral/directional dynamics of the $\mathrm{X}-33$ vehicle at critical conditions during the entry flight. By assuming that a linear relationship exists between the rotational speed vector $y$ and the actual actuators deflections, [13] formulates the problem as:

$$
\underline{y}=B \underline{\delta}
$$

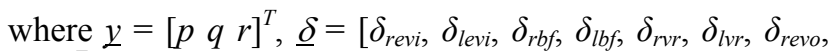
$\left.\delta_{\text {levo }}\right]^{T}$ with $\delta_{\text {revi }}, \delta_{\text {levi }}=$ right and left inboard elevons; $\delta_{r b f}, \delta_{l b f}$ $=$ right and left body flaps; $\delta_{r v r}, \delta_{l v r}=$ right and left rudders; and $\delta_{\text {revo }}, \delta_{\text {levo }}=$ right and left outboard elevons and $B$ is a $3 \times 8$ real valued matrix. Here we assume the rate limits for each actuator to be equal to $60 \mathrm{deg} / \mathrm{s}$.

Now to satisfy (30) as well as control surfaces limits such as $\delta_{i}^{\min } \leq \delta_{i} \leq \delta_{i}^{\max } i \in\{r e v i$, levi, rbf, lbf, rvr, lvr, revo, levo $\}$, we formulate a quadratic programming problem in accordance with formation $(19,20)$ by handling (30) like (13). For that, we choose the following optimality criterion at time $t+\Delta t$ :

$$
f(\underline{\delta})=\left(\underline{\delta}-\underline{\delta}_{t}\right)^{T} \Pi\left(\underline{\delta}-\underline{\delta}_{t}\right)+\gamma(\underline{y}-B \underline{\delta})^{T} \Lambda(\underline{y}-B \underline{\delta})(31)
$$

where $\Lambda$ is a diagonal matrix with diagonal positive elements $w_{p}, w_{q}, w_{r}$. $\Pi$ is a diagonal matrix with diagonal positive elements $\pi_{i} i \in I$.

\subsection{Soft fault scenario}

Here we present a simulation scenario where the proposed on line optimization algorithm is applied to perform a succession of complex roll manoeuvers as shown in Fig 2. There it is assumed that all actuators are fault free except the rate limits of left inboard elevon which changes to 20 $\mathrm{deg} / \mathrm{s}$ at $1.5 \mathrm{~s}$. The sampling time adopted by the digital control system of the different actuators is taken equal to $0.05 \mathrm{~s}$. The weights of the optimality criterion (31) are chosen as, $\gamma=10^{6}, w_{p}=10, w_{q}=1, w_{r}=1, \pi_{i}=1$, where the high value of $\gamma$ is chosen such that equality constraints such as (11) are prioritized. In Fig 2 to Fig 11, the star symbol denotes the failure instant.

From Fig 2, we can see that the algorithm reallocates actuators successfully to combine the desired angular rates. The time evolution of actuators position is shown in Fig 3. The number of iterations and error between desired and combined command signals are displayed by Fig 4 and Fig 5 respectively.

From Fig 3, the algorithm reallocates the actuators successfully and their positions are within position limits at every instant. 


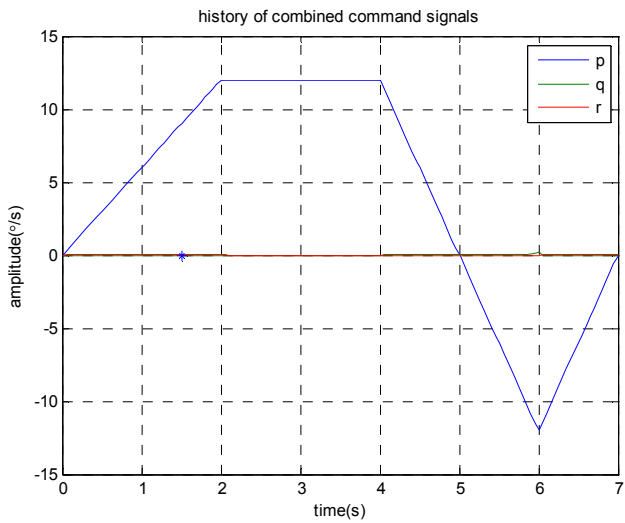

Fig 2. Time evolution of angular rates under soft fault scenario

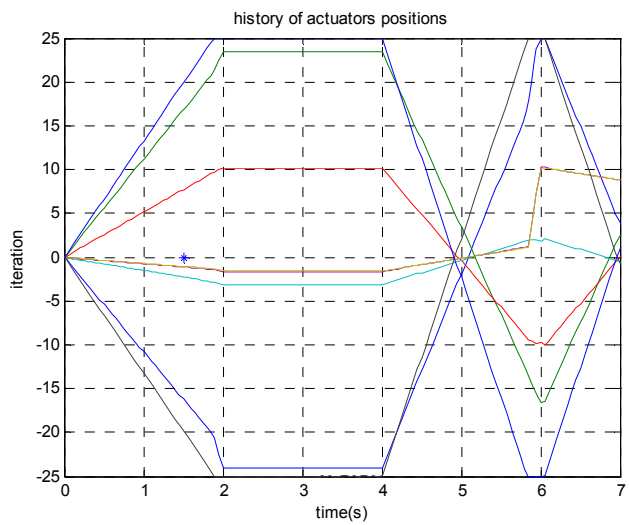

Fig 3. Time evolution of actuators positions under soft fault scenario

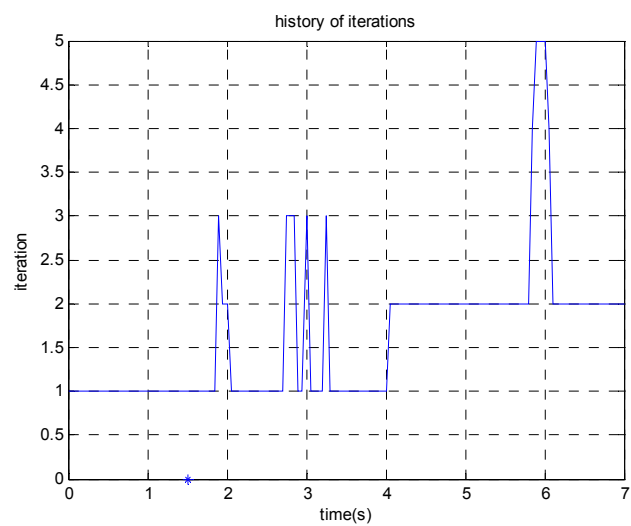

Fig 4. Number of iterations for convergence under soft fault scenario

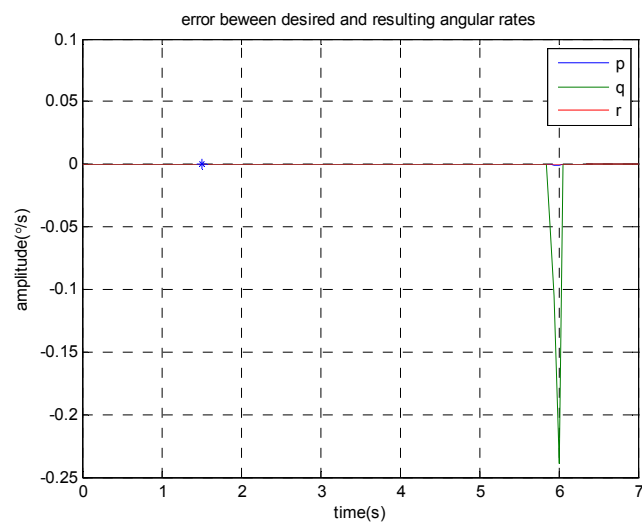

Fig 5. Angular rate errors under soft fault scenario
From Fig 4, normally the algorithm will find the optimal solution very fast except when a degraded objective should be obtained. This degradation can be seen from Fig 5, the error between combined and desired command signals can be tolerated. From (31), it is clear that the error is introduced by the penalty function and the error can be reduced by setting $\gamma$ larger. Fig 6 displays the speed of the failed actuator which reaches at different stages its speed limit.

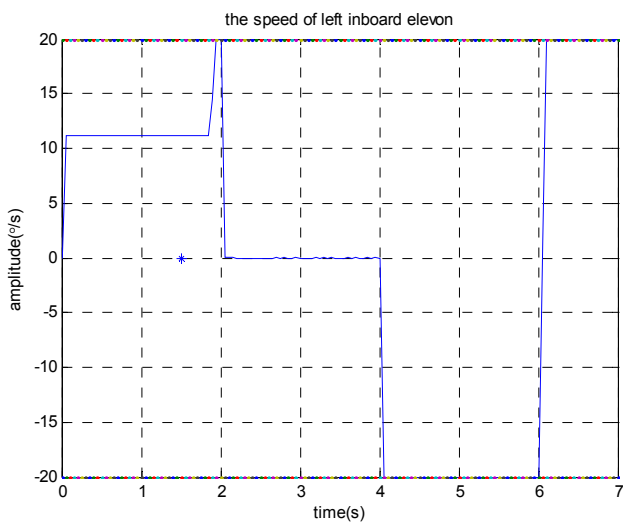

Fig 6. Example of the speed of actuator under soft fault scenario (e.g. left inboard elevon)

It appears that the active set can handle the failure situation satisfactory even if many realistic factors such as the dynamics of the actuators and dynamic inversion controller time lags where not considered.

\subsection{Hard fault scenario}

A more serious failure case occurs when an actuator remains stuck. We simulate the case where the left inboard elevon is stuck at its previous position at $1.5 \mathrm{~s}$. Simulation parameters are the same as in section 6.1, except that the maximum amplitude of angular rate $p$ is $10 \mathrm{deg} / \mathrm{s}$ for the sake of realization. The corresponding results are displayed in Fig 7 to Fig 11.

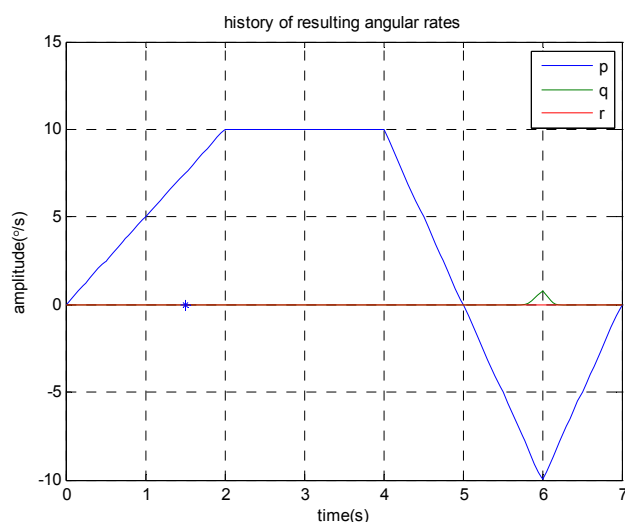

Fig 7. Time evolution of angular rates under hard fault scenario

From Fig 8 and Fig11, it can be concluded that algorithm works good to sustain the fault actuator at a fixed position after failure. From Fig 7 and Fig 10, the resulting angular rates are in the tolerance. More importantly, the algorithm finds the optimal solution very fast from Fig 9 . 


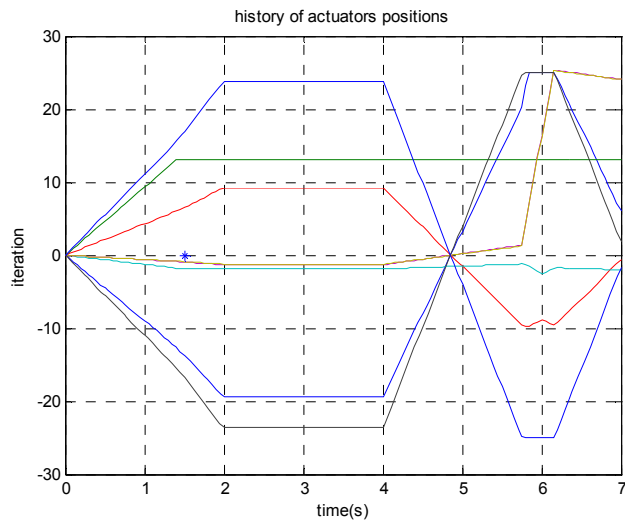

Fig 8. Time evolution of actuators positions under hard fault scenario

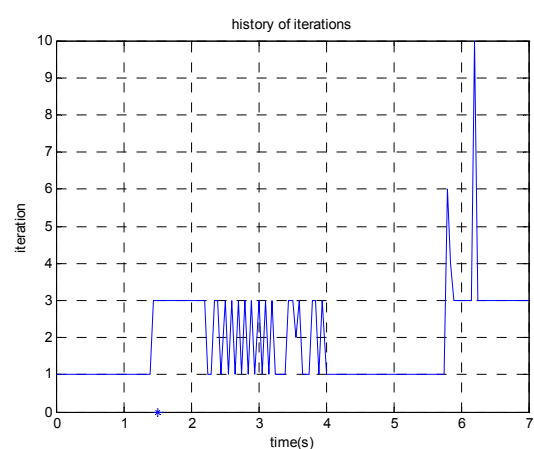

Fig 9. Number of iterations for convergence under hard fault scenario

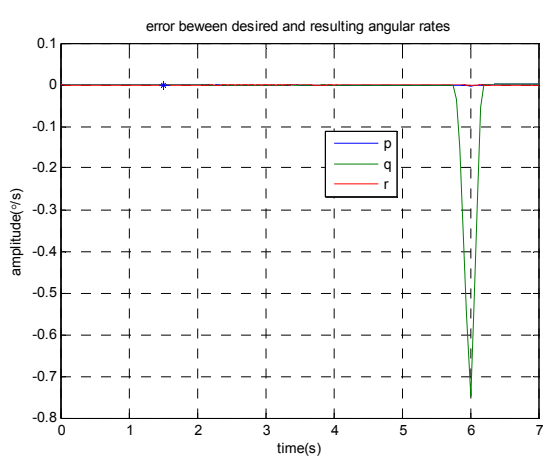

Fig 10. Error between desired and resulting angular rates under hard fault scenario

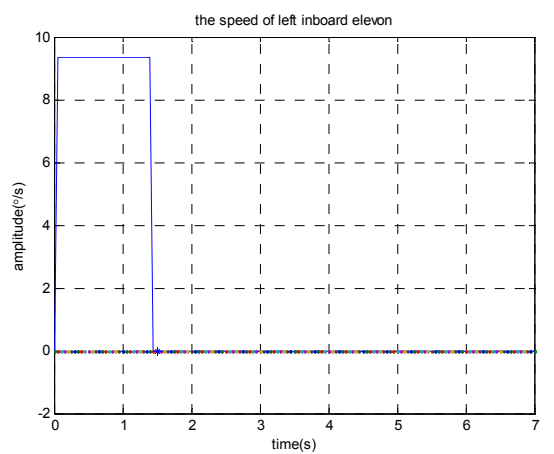

Fig 11. Example of the speed of actuator under hard fault scenario (e.g. left inboard elevon)

\section{Conclusion}

In this paper, a new approach to manage the control surfaces of an aircraft under an actuator failure scenario has been developed. The main objective has been to maintain as much as possible the maneuverability of the aircraft through the actuators while limiting the structural strain (maximum wing bending and twisting torques) of the aircraft.

This paper has shown that once the necessary aerodynamics forces and torques to perform a manoeuver have been computed by inversion of the flight dynamics, the contributions of each remaining actuator to the aerodynamic forces and torques can be optimized on-line by using an active set method which solves a linear quadratic optimization problem. Two scenarios have been considered, one where a soft failure occurs and one where a hard failure occurs, the corresponding numerical applications display the feasibility of the proposed approach.

\section{REFERENCES}

[1] M. España and G. Gilyard, Aircraft Adaptive Performance Optimization Using Redundant Control Effectors, in Automatic Control in Aerospace, Palo Alto, CA USA, 12-16, 1994.

[2] Gaulocher, S. L., Cumer, C. and Alazard, D., Aircraft load alleviation during maneuvers using optimal control surface combinations, Journal of Guidance Control and Dynamics, Vol. 30, No. 2, 591-600, 2007.

[3] Burken, J. J., Lu, P., Wu, Z. and Bahm, C., Two Reconfiguration Flight-Control Design Methods: Robust Servomechanism and Control Allocation, Journal of Guidance, Control and Dynamics, Vol. 24, No. 3, 482-494, 2001.

[4] Lunlong Zhong, Félix Mora-Camino, Neural Networks Based Aircraft Fault Tolerant Control, 12th AIAA Aviation Technology, Integration, and Operations (ATIO) Conference and 14th AIAA/ISSMO Multidisciplinary Analysis and Optimization Conference, Indiana, USA, 2012.

[5] Etkin, B. and Reid, L. D., Dynamics of flight: stability and control, 3 edition, Wiley, 1995.

[6] Roux, E. , Modèle de Masse Voilure: Avions de transport civil, Ph.D. Dissertation, SupAéro-ONERA, Toulouse, France, 2006.

[7] Andrei,G., Contribution à la commande tolérante aux pannes pour la conduite du vol, Ph.D. Dissertation, INSA, Toulouse, France, 2010.

[8] Luenberger, D. G. and Ye, Y., Linear and Nonlinear Programming. Springer, 2008.

[9] Nocedal, J. and Wright, S. J., Numerical Optimization, Springer, 1999.

[10] H. W. Kuhn, A. W. Tucker, Nonlinear programming, in Proceedings of 2nd Berkeley Symposium, Berkeley, 481-492, 1951.

[11] Boyd, Stephen, Vandenberghe, Lieven, Convex Optimization, Cambridge University Press, 2004.

[12] Harkegard, O., Efficient active set algorithms for solving constrained least squares problems in aircraft control allocation, in the 41st IEEE Conference on Decision and Control, 1295-1300, 2002.

[13] M. Demenkov, Geometric Algorithms for Input Constrained Systems with Application to Flight Control, Ph.D. Dissertation, De Montfort University, UK, 2007. 